\title{
Case Studies of Statistical Entropy Analysis in Recycling Processes: A Tool in Support of a Circular Economy ${ }^{\dagger}$
}

\author{
Omar Velazquez Martinez ${ }^{1}$, Annukka Santasalo-Aarnio ${ }^{1}$, Markus A. Reuter ${ }^{2}$ and Rodrigo Serna- \\ Guerrero $^{1}$ \\ 1 Aalto University, Espoo, Finland \\ 2 Helmholtz Institute Freiberg for Resource Technology, Freiberg, Germany \\ + Presented at the Entropy 2021: The Scientific Tool of the 21st Century, 5-7 May 2021; Available online: \\ https://sciforum.net/conference/Entropy2021/.
}

Published: 5 May 2021

Statistical entropy (SE), has been used alongside material flow analysis (MFA) to aid studies of resource efficiency and waste management. Given that statistical entropy operates based on principles of information theory, it can be used to describe quantitatively systems with mixed materials flow, including their overall distribution of components throughout transformational stages, e.g., mechanical pre-treatment stages in recycling processes. In the present work, we present two cases where our research group has used statistical entropy analysis as an analytical tool for recycling process, with the aim of supporting a transition towards a circular economy.

In the first place, a comparison between two lithium-ion battery recycling processes was carried out by the combination of material flow analysis and statistical entropy. In this manner, an efficiency weight at a systemic level is given to the mechanical processing stages, which is reflected by the entropic values at final stages. In other words, while both systems obtained recycled materials with similar characteristics, the system that presented a more efficient pre-processing stage (i.e., lower statistical entropy value) presented a lower entropy value of the substances at final stage. Secondly, statistical entropy and material flow analysis was used as to aid in the design of mechanical processing stages for thermoelectric devices (TEDs). A total of 106 thermoelectric devices were mechanically processed by different comminution methods, physical and chemical characterization were carried out and the entropy values evaluated in each stage. The statistical entropy results were used to design a process which presented a fast and efficient entropy reduction in all the throughput, such, entailed a total of 5 stages and obtained fractions of high purity and treatability.

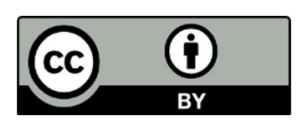

(C) 2021 by the authors. Licensee MDPI, Basel, Switzerland. This article is an open access article distributed under the terms and conditions of the Creative Commons Attribution (CC BY) license (http://creativecommons.org/licenses/by/4.0/). 